\title{
Auditory Temporal Processing and Working Memory: Two Independent Deficits for Dyslexia
}

\author{
Leah Fostick \\ Ariel University Center, \\ Ariel, Israel
}

\author{
Sharona Bar-El, Ronit Ram-Tsur \\ Bar-Ilan University, \\ Ramat-Gan, Israel
}

\begin{abstract}
Dyslexia is a neuro-cognitive disorder with a strong genetic basis, characterized by a difficulty in acquiring reading skills. Several hypotheses have been suggested in an attempt to explain the origin of dyslexia, among which some have suggested that dyslexic readers might have a deficit in auditory temporal processing, while others hypothesized that dyslexia origins from a deficit in working memory. The current study was designed to test whether working memory and/or auditory temporal processing can predict reading ability in normal and dyslexic readers. Fifty-three adults were diagnosed with phonological dyslexia and 46 normal reading adults were tested on reading regular words, auditory temporal order judgment, and backward digit span. The results are that: For dyslexic readers, both auditory temporal processing and working memory are correlated with reading, even after controlling for their covariance. However, no correlation between reading measures, temporal processing and working memory are found for normal readers. The conclusions are that: (1) Both auditory temporal processing and working memory are significantly involved in reading among dyslexic readers; and (2) Dyslexic readers might use more different reading strategies than normal readers do, who apparently are not involved in auditory temporal processing and working memory while reading.
\end{abstract}

Keywords: auditory temporal processing, temporal order judgment, working memory, dyslexia, language

\section{Introduction}

Developmental dyslexia, also known as specific reading disability, is a neuro-cognitive disorder with a strong genetic basis, characterized by a difficulty in acquiring reading skills, in spite of normal intelligence and sufficient reading opportunities. Developmental dyslexia affects about $5 \%$ to $10 \%$ of the population (Shaywitz, 1996). Over the past decades, several different hypotheses have been presented to explain the origin of dyslexia. The classic, phonological hypothesis posits that dyslexia represents a difficulty in learning and store the relations between letters and their matching sounds. A number of studies have reported that dyslexic readers have difficulties in reading regular and non-words, spelling, and manipulating speech sounds (Ahissar, Protopapas, Reid, \& Merzenich, 2000; Ben-Artzi, Fostick, \& Babkoff, 2005; Ramus et al., 2003; Snowling, Bishop, \& Stothard, 2000), although they have normal intelligence and reading comprehension (Ben-Artzi, et al., 2005; Ramus et al., 2003; Snowling, 2001). However, the phonological hypothesis limits the origin of the deficit in dyslexia to the ability to process speech sounds, while other

Leah Fostick, Ph.D., Department of Communication Disorders, Ariel University Center.

Sharona Bar-El, School of Education, Bar-Ilan University.

Ronit Ram-Tsur, School of Education, Bar-Ilan University. 
theories have shown dyslexic readers to have other perceptual and cognitive difficulties which are also reflected in their lower ability to read.

In addition to phonological difficulties, additional difficulties in the auditory domain have been reported among dyslexic readers, e.g., difficulties in frequency and amplitude discrimination (Amitay, Ahissar, \& Nelken, 2002; Banai \& Ahissar, 2004; France et al., 2002; Goswami et al., 2002), and auditory temporal resolution (Ben-Artzi et al., 2005; Tallal, 1980). The latter findings have led to the second hypothesis, the auditory temporal resolution hypothesis, originally was suggested almost 40 years ago by Tallal and his colleagues (Tallal, 1980; Tallal \& Piercy, 1973a, 1973b). This hypothesis posits that reading impairment is caused by a fundamental perceptual deficit in processing rapid, auditory, or visual stimuli (Ben-Artzi et al., 2005; Ram-Tsur, Faust, \& Zivotofsky, 2006, 2008; Tallal, 1980). According to this model, speech, which is composed of brief stimuli presented rapidly, is especially vulnerable to deficit in temporal processing since this impairment reduces the ability of the individual to perceive critical elements in the speech stream accurately. This inability, in turn, disrupts the establishment of a stable phonological code (Ben-Artzi et al., 2005; Meyler \& Breznitz, 2005; Tallal \& Piercy, 1973b).

The auditory temporal resolution hypothesis predicts that reading impaired children would have difficulty in processing linguistic, as well as non-linguistic stimuli. Studies conducted by Tallal and others (Ben-Artzi et al., 2005; Ramus et al., 2003; Reed, 1989; Tallal, 1980; Tallal \& Piercy, 1973a, 1973b) indeed show that reading impaired children have difficulties in identifying the temporal order of two stimuli when they are presented rapidly, but not when they are presented at a slower rates (Ben-Artzi et al., 2005; Breier et al., 2001). Since Tallal's classical experiments about the difficulties in temporal processing among dyslexic readers have been reported by a number of researchers using a variety of different paradigms of temporal resolution or acuity: (1) backward masking (Ramus et al., 2003); (2) gap detection (Van Ingelghem et al., 2001); and (3) categorical perception of phonemes and non-speech analogues (Breier et al., 2001; Reed, 1989; Serniclaes, Sprenger-Charolles, Carre, \& Demonet, 2001).

Another hypothesis attempting to explain the origin of developmental dyslexia, suggests that a deficit in working memory underlies reading difficulties in dyslexia, at least for a subset of dyslexic reader who is found to have poor working memory, along with poor frequency and duration discrimination (Banai \& Ahissar, 2004; Shankweiler \& Crain, 1986). Working memory refers to the ability to process and manipulate information while being received, in order to use it for continuous behavior (Baddeley, 2003). Baddely hypothesized that working memory includes two systems of encoding sound-based (the phonological loop) and visual- and spatial-based information (the visuo-spatial sketchpad), as well as a generating system (the central executive). This latter system integrates the information received through the phonological loop and the visuo-spatial sketchpad, with already stored information, while also controlling and monitoring attention. According to the hypothesis, the primary function of phonological short-term memory is to support the long-term learning of the phonological structure of the language (Baddeley, Gathercole, \& Papagno, 1998; Gathercole, Tiffany, Briscoe, \& Thorn, 2005). Therefore, inadequate short-term memory will cause difficulties in learning the sound structure of new words (Gathercole et al., 2005). In an attempt to understand the nature of working memory deficit among dyslexic readers, Jeffries and Everatt (2004) tested dyslexic children on a battery of tasks and found that the dyslexic readers are worse than the normal readers in the phonological loop and the central executive, except in the visuo-spatial sketchpad. Based on the similar findings, Archibald and Gathercole (2006) established the "double-jeopardy" hypothesis for two independent deficits in working memory among children 
with language impairment: One is in the phonological loop and the other is in the central executive.

Taken together, both the auditory temporal resolution deficit hypothesis and the working memory deficit hypothesis for dyslexia have competing suggestions for the origin of dyslexia that can explain the phonological deficits shown by dyslexic readers. Moreover, some researchers suggested that working memory underlies the difficulties that dyslexic readers exhibit in auditory temporal resolution tasks, since it reduces the access to stored information (Banai \& Ahissar, 2004). Several questions remain to be addressed regarding the reported deficits of dyslexic readers in working memory and auditory temporal resolution. Firstly, to date, no study has actually tested the same dyslexic readers on both working memory and auditory temporal resolution to verify or reject the hypothesis that the deficit in auditory resolution is secondary to the deficit in working memory. Secondly, to date, most studies have compared groups of normal readers with dyslexic readers on a number of measures. There are few, if any, studies that have been designed to test whether deficit in working memory and/or auditory temporal resolution can significantly predict deficit in reading performance. Therefore, the current study was designed to: (1) evaluate both working memory and auditory temporal resolution in the same normal adult and dyslexic readers; and (2) test whether working memory and/or auditory temporal resolution can predict reading ability in normal and dyslexic readers.

\section{Methods}

\section{Participants}

Fifty-three adults were diagnosed with dyslexia (32 men, 21 women, mean age $=26.45, S D=4.14$ ), and 46 normal readers adults (30 men, 16 women, mean age $=24, S D=2.84$ ), participated in the study. The groups were similar in their educational status, but dyslexic readers were significantly older than the normal readers ( $p$ $<0.01$ ). Diagnosis of dyslexia was based on having official diagnosis from a known diagnostic authority. Additional diagnostic tests that were conducted prior to participating in the study demonstrated poor phonological awareness among the dyslexic groups (see Table 1). All participants were screened for normal hearing, and were native Hebrew speaking university or college students.

Table 1

Means and SDs of Dyslexia Diagnostic Tests for Dyslexic and Normal Readers

\begin{tabular}{|c|c|c|c|c|c|}
\hline \multirow{2}{*}{ Variable } & \multicolumn{2}{|c|}{ Dyslexic reader } & \multicolumn{2}{|c|}{ Normal reader } & \multirow{2}{*}{$F$} \\
\hline & M & $S D$ & M & $S D$ & \\
\hline Phoneme deletion & 16.08 & 3.37 & 19.43 & 0.96 & $42.72^{* * *}$ \\
\hline Spoonerism & 3.60 & 1.76 & 5.52 & 0.72 & $47.72^{* * *}$ \\
\hline Pig Latin & 3.43 & 2.35 & 5.09 & 1.49 & $16.88^{* * *}$ \\
\hline
\end{tabular}

Note. ${ }^{* * *} p<0.001$.

\section{Reading: Regular Words}

Hebrew written language has both deep and shallow orthographies. In shallow orthography, the words are pointed creating a high spelling-to-sound correspondence, and in deep orthography, the words are un-pointed, creating a low spelling-to-sound correspondence (Frost, 1994). To evaluate reading skills, the authors used reading regular, un-pointed words (Shatil, 1995b, for a detailed explanation about punctuation in Hebrew, see Ram-Tsur, Faust, \& Zivotofsky, 2008). This task was designed to measure the amount of correct words that the participant is able to read per minute. The participants were presented with a list of 
217 solitary un-pointed words. The words included low and high frequency words, words with different lengths (three to seven letters), verbs and nouns in different declensions, and homographs (i.e., words that can be read differently without punctuation). Each participant was asked to read the words as fast and correctly as he/she can during one minute. The score reflects the number of words per minute the participant read correctly (Shatil, 1995b).

\section{Temporal Processing: Dichotic TOJ (Temporal Order Judgment)}

Subjects were presented with a pair of 15 msec duration $1.8 \mathrm{kHz}$ tones presented dichotically (the first tone to one ear the second tone to the other ear), and were required to reproduce the order in which they heard the tones (left first then right; or right first then left). Tone combinations were presented in a random order with ISI (inter-stimulus interval) $=5,10,15,30,60,90,120$, or 240 msec. This task minimized the use and need for the working memory, since the subject responds to a single pair of stimuli that are separated by no greater than 240 msec. The order of the presentation of ISIs was also random. Each ISI value was repeated 16 times, resulting in a total of 256 trials. After every 32 trials, subjects received a short recess. Percent correct was recorded for each participant for each ISI, and the threshold was obtained as the ISI for $75 \%$ correct.

Prior to the experiment, participants were required to fulfill a training phase. To familiarize the participants with the tones, participants were first presented with six examples of the tone in one ear, then six examples of the tone in the other ear. Training then proceeded with 24 trials, 12 tones in each ear, randomly intermixed. On each trial, the participants were required to identify the sound location by pressing the correct key. Visual feedback ("Right”/“Wrong”) was provided for each response. In the last stage of the familiarization phase, the stimuli were presented in random order, with no feedback, until the participants met the criterion of 20 correct responses in 24 consecutive trials. Participants who were successful in the familiarization phase, were then presented with 16 pairs of stimuli in two possible patterns: left-right, right-left, with ISIs of 240 and $60 \mathrm{msec}$, resulting in 64 pairs of stimuli. Participants were to identify which pattern they heard by pressing the key for the first sound followed by the key for the second sound. Visual feedback was provided on all training trials. No feedback was provided during the experimental session (Ben-Artzi et al., 2005).

\section{Working Memory}

Working memory was measured using the WAIS-III (Wechsler Adults Intelligence Scale version III, Wechsler, 1997) subtest for backward digit span. In this subtest, the experimenter reads aloud lists of digits at a rate of one digit per second. Immediately after the set of digits had been read, participants were instructed to report back the digits verbally in the reverse order they heard it. Participants received two trials at each set size starting at set size 2 and working up to set size 8 . Testing was terminated when participants were incorrect on both trials of a given set size. The digit backward score was the number of digit backward trials where all digits were reported accurately in the correct reverse order (maximum score of 14) (Wechsler, 1997). Backward digit span is a subtask of the digit span subtest, and is used as an indicator for the working memory (Gathercole \& Pickering, 2000).

\section{Apparatus}

Psychophysical tasks were presented using a Pentium1 personal computer that controlled the stimulus presentation, recorded responses and response time. Auditory stimuli used in the psychophysical tasks were generated by a sound-generator device (TDT-system II: Tucker-Davis Technologies, Gainesville, FL), and then 
presented binaurally through TDH-49 headphones. Tasks were programmed using Matlab ${ }^{\mathrm{TM}}$ software version 6.5 .

Screening for hearing sensitivity was performed using Danplex DA64 or MA32 audiometers (Maico Hearing Instruments Ltd.).

\section{Procedure}

The results reported in the current study are a part of larger study including a large battery of psychophysical, lingual, and cognitive tasks. The experiment was carried out in three sessions, approximately for two hours each. Two sessions included the psychophysical and cognitive tasks in random order, and one session included all the lingual tasks. Order of sessions across participants was fully counterbalanced. Prior to the experiment, subjects received full explanation about the study, signed an informed consent, and were screened for normal hearing. Participants were paid an amount in NIS, equivalent to $\$ 75$ for completing the entire study. The study was approved by the Bar-Ilan University Institutional Human Studies Review Board.

\section{Results}

\section{Reading, Working Memory, and Temporal Processing for Dyslexic and Normal Readers}

Table 2 presents descriptive and inferential statistics of reading, dichotic TOJ thresholds and digit span for dyslexic, and normal readers. As can be seen, dyslexic readers showed poorer reading and working memory scores and higher dichotic TOJ thresholds.

Table 2

Means and SDs of Reading Variables, Dichotic and Gap Detection Thresholds and Digit Span for Dyslexic and Normal Readers

\begin{tabular}{|c|c|c|c|c|c|c|c|c|c|}
\hline \multirow{2}{*}{ Variable } & \multicolumn{4}{|c|}{ Dyslexic reader } & \multicolumn{4}{|c|}{ Normal reader } & \multirow{2}{*}{$F$} \\
\hline & $M$ & $S D$ & Skew & SE skew & $M$ & $S D$ & Skew & SE skew & \\
\hline Regular words $^{\mathrm{a}}$ & 80.75 & 26.83 & -0.26 & 0.33 & 125.07 & 15.49 & 0.33 & 0.35 & $97.26^{* * *}$ \\
\hline Dichotic TOJ $^{\mathrm{b}}$ & 126.58 & 77.39 & 0.78 & 0.35 & 74.42 & 58.86 & 1.98 & 0.35 & $13.03^{* * *}$ \\
\hline Sqrt dichotic TOJ ${ }^{\mathrm{b}}$ & 10.13 & 2.94 & -0.02 & 0.37 & 7.71 & 2.49 & 0.40 & 0.36 & $16.82^{* * *}$ \\
\hline Backward digit span & 6.34 & 1.92 & 0.42 & 0.33 & 8.07 & 2.31 & 0.20 & 0.35 & $16.44^{* * *}$ \\
\hline
\end{tabular}

Notes. ${ }^{\mathrm{a}}$ Number of words read correctly; ${ }^{\mathrm{b}}$ in msec; ${ }^{* * *} p<0.001$.

\section{Reading Ability and Temporal Processing/Working Memory}

Figures 1 and 2 present the associations between digit span and dichotic TOJ, and the two reading variables. As can be seen, although the correlation with reading was significant both with dichotic TOJ ( $r=$ -0.49, $p<0.001)$ and with backward digit span $(r=0.38, p<0.001)$, the correlation patterns were different for dyslexic and normal readers. Therefore, separate correlation analyses for each group were performed. The results showed that even for normal readers, neither dichotic TOJ nor digit span was significantly correlated with reading ability ( $r=-0.13, p>0.05$; and $r=-0.04, p>0.05$, respectively), both of them were associated with reading ability for the dyslexic readers ( $r=-0.38, p<0.05$; and $r=0.31, p<0.05$, respectively).

In order to examine the unique contribution of temporal processing to reading capacity, beyond working memory ability, for dyslexic and normal readers, a multiple regression was conducted for dyslexic readers' data, with the number of words read correctly as the predicted variable. Table 3 shows that both digit span and dichotic TOJ threshold were significant predictors for regular words reading. 


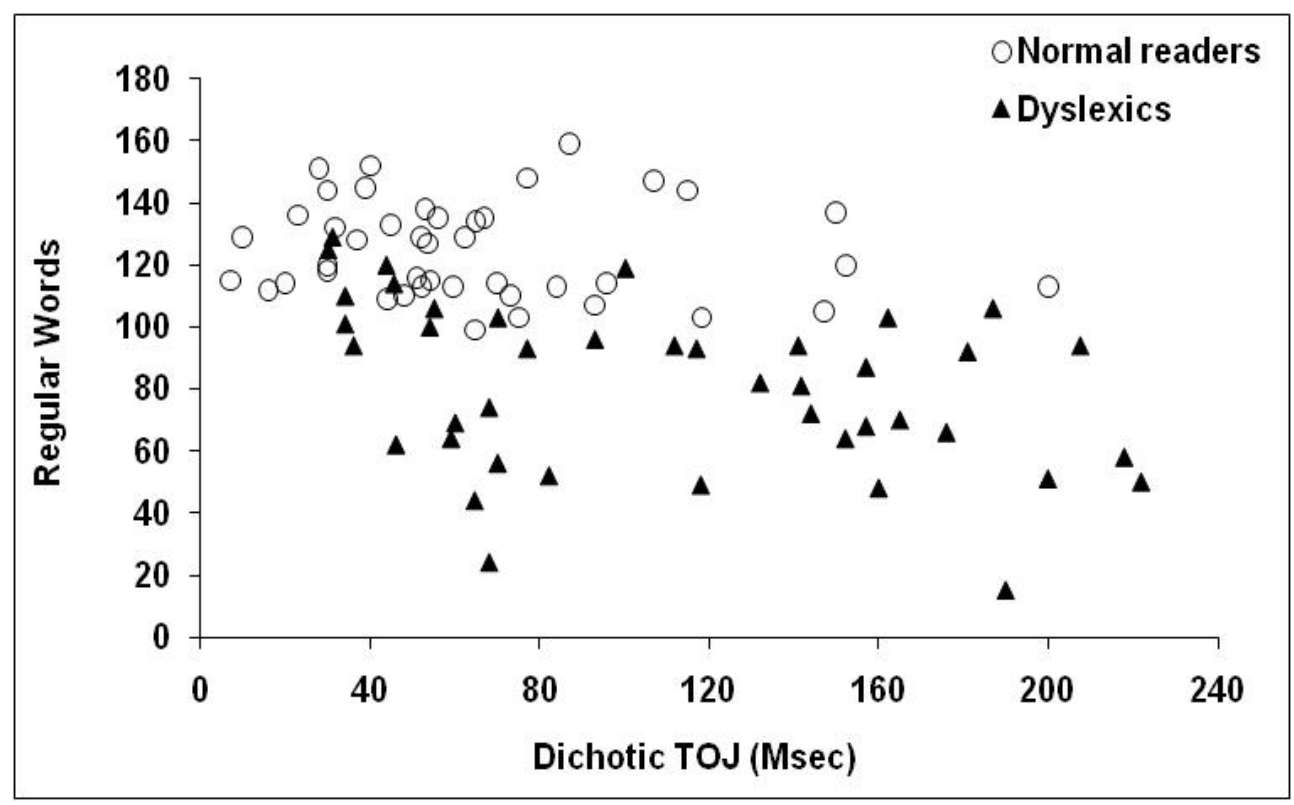

Figure 1. Reading regular words by dichotic TOJ for dyslexic and normal readers.

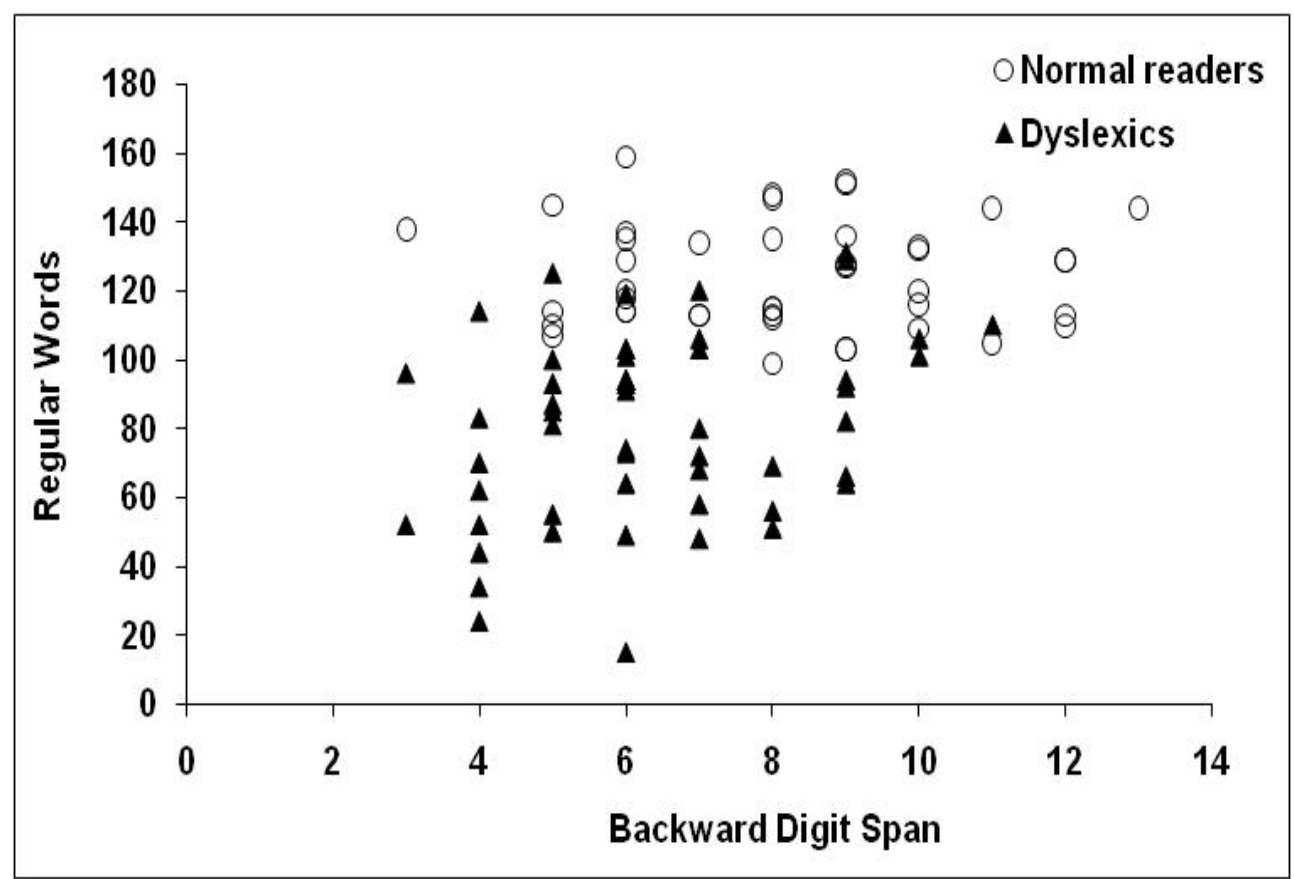

Figure 2. Reading by digit span for dyslexic and normal readers.

Table 3

Multiple Regression Predicting Number of Regular Words Correctly Read by Backwards Digit Span and Dichotic TOJ

\begin{tabular}{lclllll}
\hline Variable & Beta & $t$ & $R^{2}$ & $F$ & $R^{2}$ change & $F$ change \\
\hline Backwards digit span & 0.34 & $3.24^{* *}$ & 0.11 & $10.47^{* *}$ & 0.11 & $10.47^{* *}$ \\
Dichotic TOJ & -0.24 & $2.90^{* *}$ & & & & \\
\hline
\end{tabular}




\section{Discussion}

Adult dyslexic readers were significantly poorer than normal readers in both the digit span test and dichotic temporal order judgments. Furthermore, among the dyslexic readers, reading ability could be significantly predicted by their performance on working memory and on dichotic TOJ tasks, supporting these abilities as two independent factors that influence reading skills. However, for the normal reader, their performances on working memory and on dichotic TOJ were not related to reading ability.

Findings of auditory temporal resolution deficit in children with dyslexia and in adult dyslexic readers have been reported for the past four decades. The first reports were published by Tallal in what is considered now as classic studies, in which subjects with reading and language impairments were required to reproduce the order of two high and low frequency brief tones, and made significantly more TOJ errors than normal readers when tones' duration was relatively short $(75 \mathrm{~ms})$, rather than long $(250 \mathrm{~ms})$, and/or when the ISI was relatively short $(150 \mathrm{~ms})$, rather than long (300 ms) (Tallal, 1980; Tallal \& Piercy, 1973a). Tallal's results were replicated by a number of other researchers who used similar and other methods and showed that dyslexic readers need longer ISIs to reproduce the order of two tones, and that the ability is related to their reading and phonological ability (Ahissar et al., 2000; Ben-Artzi et al., 2005; Breier et al., 2001; Ramus et al., 2003; Reed, 1989). However, these findings were not replicated by others (Adlard \& Hazan, 1998; Hill, Bailey, Griffiths, \& Snowling, 1999; Mody, Studdert-Kennedy, \& Brady, 1997; Snowling, 2001). According to Tallal et al. (Tallal, 1980; Tallal \& Piercy, 1973a, 1973b), dyslexic readers should have greater difficulty than normal readers in discriminating the temporal order when the stimuli are separated by short ISIs. However, a number of studies have reported the data that do not support this prediction. For example, Mody, Studdert-Kennedy, and Brady (1997) found no difference between dyslexic and normal readers in reproducing the order of speech stimuli (/ba/-/sa/ or $\left./ \mathrm{da} /-/ \int \mathrm{a} /\right)$, when the stimuli were separated by short ISIs (10 to $100 \mathrm{msec}$ ). Although Marshall et al. (2001) did find that dyslexic readers were poorer than normal readers in reproducing the order of two 100 and $305 \mathrm{~Hz}$ tones, they did not find a group $\times$ ISI interaction, thus contradicting Tallal's prediction that dyslexic readers would have a TOJ deficit when the stimuli are separated only by short ISIs. Supporting the TOJ deficit hypothesis, in the current study, the authors found that dyslexic readers' auditory temporal resolution threshold, as measured by dichotic TOJ, was significantly longer than the threshold of normal readers. These findings support previous studies showing an auditory temporal resolution deficit in dyslexia.

Based on the need for an adequate verbal working memory in order to read properly, several studies have examined working memory among dyslexic readers and have reported deficient verbal working memory in dyslexia (Banai \& Ahissar, 2006; Brambati et al., 2006; Gathercole \& Pickering, 2000; Jeffries \& Everatt, 2004; Ram-Tsur et al., 2006, 2008). In the current study, the authors also found that the working memory of dyslexic readers, as measured by the backwards digit span task, was significantly poorer than that of normal readers. These findings support the hypothesis of poorer working memory in a population of adult dyslexic readers.

The main symptom of phonological dyslexia is poor phonological processing which is reflected in a difficulty of the dyslexic readers in analyzing, synthesizing and manipulating speech sounds (Snowling et al., 2000). Along his line, studies have shown poor basic phonological abilities among dyslexic readers, which, in turn gave rise to the hypothesis that the dyslexic readers were deficient only in phonological processing, but not 
in processing non phonological, auditory material (Mody et al., 1997; Snowling et al., 2000; Vellutino, Fletcher, Snowling, \& Scanlon, 2004). In the current study, the authors found, as expected, poor reading and phonological processing among dyslexic readers. However, the authors also found that working memory and auditory temporal resolution were not only poorer among dyslexic readers, but also were independently associated with their reading ability. Thus, it appears that both working memory and auditory temporal resolution are basic abilities that are involved in reading among dyslexic readers (Banai \& Ahissar, 2004; Ben-Artzi et al., 2005; Reed, 1989; Tallal, 1980; Wright, Bowen, \& Zecker, 2000). The complementary finding, which among the normal readers' performances on a digit span task and on a dichotic TOJ task did not predict reading ability, leads to the suggestion that working memory and auditory temporal resolution are utilized by the dyslexic in the processing associated with reading in a manner that is not used by the normal readers. The lack of significant prediction of working memory and auditory temporal resolution among the normal readers for their reading ability cannot be attributed to a ceiling effect, i.e., to a shortened range neither in the digit span test nor in the dichotic TOJ task, since both distributions are normally distributed after the measures were transformed for the analysis. The immediate use of working memory and auditory temporal resolution in the processing of reading by the dyslexic readers and the absence of their use among the normal readers, therefore, suggests that the neurological mechanisms utilized by the normal readers are unavailable for use by the dyslexic readers. Similar findings were reported by Marshall et al. (2001) when he tested young normal readers aged six to 13 on auditory repetition test and on reading measures. Performance on their auditory repetition test was not related to reading measures when controlled for age and IQ (intelligence quotient).

The present findings indicate that not only are dyslexic readers deficient as a group both in working memory and in auditory temporal resolution when compared with the normal reader, but also within the dyslexic group, the ability of the individual dyslexic to read, is significantly predicted by their performance on digit span and on dichotic TOJ. Although each of the two cognitive functions, i.e., working memory and auditory temporal resolution, was deficient among dyslexic readers and was related to their reading ability, the analysis indicated that working memory and auditory temporal resolution are not significantly correlated. These findings imply that the deficiency in working memory and the deficiency in auditory temporal resolution are two independent sources of difficulty for the dyslexic readers in processing written material. Two independent deficits, each of which predicts reading ability in a population of dyslexic readers, may help explain the large inter-individual differences found in this population (Ramus et al., 2003; Reid, Szczerbinski, Iskierka-Kasperek, \& Hansen, 2007; Wright et al., 2000). Similarly, while addressing the issue of the relatively large inter-individual differences found among dyslexic readers, Wright et al. (2000) suggested that reading problems are in fact multidimensional in nature. According to these and other investigators (Ramus et al., 2003; Reid et al., 2007), dyslexia may not be the result of a sole deficit, but rather, the product of several degraded processes. They suggested that the deficits may be in both the auditory and the visual processing of written material.

As noted above, the present findings support a conclusion that the dyslexic readers and the normal readers use different neuropsychological mechanisms to read. Similar conclusions were reached in a number of imaging studies that concluded that dyslexic readers were utilizing more different regions of the brain than normal readers did, while reading. The imaging literature is not consistent in identifying the specific brain regions used by the normal readers and by the dyslexic readers. Nevertheless, the pattern of all these studies seems to indicate the use of different brain regions by the normal readers and the dyslexic readers while 
performing a reading task. For example, Conway et al. (2008) found dyslexic readers who had greater activation in auditory cortex than the normal readers did, while performing auditory working memory tasks. Brambati et al. (2006) found less activation in the posterior temporal cortical regions of dyslexic readers than that normal readers while reading. According to Silani et al. (2005), the decrease in activation seems to be related to the altered density of grey and white matter in this region. Temple (2002) reported that dyslexic readers had decreased activation in the left temporo-parietal cortex in response to phonological stimuli and in left prefrontal cortex in response to rapid auditory stimuli. Brosnan et al. (2002) showed that dyslexic readers had less activation than normal readers in pre-frontal regions, while performing tasks that are considered to be dependent on pre-frontal processes. Vasic et al. (2008) suggested that hyper activation in other regions (for example, inferior frontal regions) may be caused by an attempt to compensate for the decrease in other regions (for example, left parieto-temporal and occipito-temporal regions). In summary, when there does not seem to be any consistency among the various studies, as to the specific brain regions involved in reading by normal readers and by dyslexic readers, all of the studies appear to conclude that the two populations use different brain regions to perform reading-based tasks.

Several questions may be raised regarding the present findings. The data which indicated that reading ability by the dyslexic readers can be predicted by the performance on working memory and on auditory temporal resolution, whereas the reading ability of the normal readers cannot be so predicted, were generated by adult normal readers and adult dyslexic readers. The question may then be legitimately raised as to whether the same difference would be found whether the comparison between the very young, beginning normal readers versus dyslexic readers or not. Perhaps all readers, both normal readers and dyslexic readers begin reading by using neuro-psychological mechanisms that are dependent upon working memory and auditory temporal resolution and only begin to differ when the reading skills become well-established. The second question that may be raised is whether there are circumstances or conditions that would also cause the normal adult readers to use and be dependent upon working memory and temporal resolution similar to the adult dyslexic readers (e.g., learning to read a foreign language), so that the reading ability of the normal readers would also be predicted by working memory and auditory temporal resolution.

\section{References}

Adlard, A., \& Hazan, V. (1998). Speech perception in children with specific reading difficulties (dyslexia). Quarterly Journal of Experimental Psychology Section A-Human Experimental Psychology, 51(1), 153-177.

Ahissar, M. (2007). Dyslexia and the anchoring-deficit hypothesis. Trends in Cognitive Sciences, 11(11), 458-465. doi: 10.1016/j.tics.2007.08.015

Ahissar, M., Protopapas, A., Reid, M., \& Merzenich, M. M. (2000). Auditory processing parallels reading abilities in adults. Proceedings of the National Academy of Sciences of the United States of America, 97(12), 6832-6837.

Amitay, S., Ahissar, M., \& Nelken, I. (2002). Auditory processing deficits in reading disabled adults. Journal of the Association for Research in Otolaryngology, 3(3), 302-320.

Archibald, L. M., \& Gathercole, S. E. (2006). Short-term and working memory in specific language impairment. International Journal of Language and Communication Disorders, 41(6), 675-693.

Baddeley, A. (2003). Working memory and language: An overview. Journal of Communication Disorders, 36(3), 189-208.

Baddeley, A., Gathercole, S., \& Papagno, C. (1998). The phonological loop as a language learning device. Psychological Review, 105(1), 158-173.

Banai, K., \& Ahissar, M. (2004). Poor frequency discrimination probes dyslexics with particularly impaired working memory. Audiology and Neurotology, 9(6), 328-340. 
Banai, K., \& Ahissar, M. (2006). Auditory processing deficits in dyslexia: Task or stimulus related? Cereb Cortex, 16(12), 1718-1728.

Ben-Artzi, E., Fostick, L., \& Babkoff, H. (2005). Deficits in temporal-order judgments in dyslexia: Evidence from diotic stimuli differing spectrally and from dichotic stimuli differing only by perceived location. Neuropsychologia, 43(5), 714-723.

Brambati, S. M., Termine, C., Ruffino, ... Perani, D. (2006). Neuropsychological deficits and neural dysfunction in familial dyslexia. Brain Research, 1113(1), 174-185.

Breier, J. I., Gray, L., Fletcher, J. M., Diehl, R. L., Klaas, P., Foorman, B. R., \& Molis, M. R. (2001). Perception of voice and tone onset time continua in children with dyslexia with and without attention deficit/hyperactivity disorder. Journal of Experimental Child Psychology, 80(3), 245-270.

Briscoe, J., \& Rankin, P. M. (2009). Exploration of a “double-jeopardy” hypothesis within working memory profiles for children with specific language impairment. International Journal of Language and Communication Disorders, 44(2), 236 - 250.

Conway, T., Heilman, K. M., Gopinath, K., ... Crosson, B. (2008). Neural substrates related to auditory working memory comparisons in dyslexia: An fMRI study. Journal of the International Neuropsychological Society, 14(4), 629-639.

France, S. J., Rosner, B. S., Hansen, P. C., Calvin, C., Talcott, J. B., Richardson, A. J., \& Stein, J. F. (2002). Auditory frequency discrimination in adult developmental dyslexics. Perception and Psychophysics, 64(2), 169-179.

Frost, R. (1994). Prelexical and postlexical strategies in reading: Evidence from a deep and a shallow orthography. Journal of Experimental Psychology: Learning, Memory, and Cognition, 20(1), 116-129.

Gathercole, S. E., Alloway, T. P., Willis, C., \& Adams, A. M. (2006). Working memory in children with reading disabilities. Journal of Experimental Child Psychology, 93(3), 265-281.

Gathercole, S. E., \& Pickering, S. J. (2000). Working memory deficits in children with low achievements in the national curriculum at 7 years of age. British Journal of Educational Psychology, 70 (Pt 2), 177-194.

Gathercole, S. E., Tiffany, C., Briscoe, J., \& Thorn, A. (2005). Developmental consequences of poor phonological short-term memory function in childhood: a longitudinal study. Journal of Child Psychology and Psychiatry, 46(6), 598-611.

Goswami, U., Thomson, J., Richardson, U., Stainthorp, R., Hughes, D., Rosen, S., et al. (2002). Amplitude envelope onsets and developmental dyslexia: A new hypothesis. Proceedings of the National Academy of Sciences of the United States of America, 99(16), 10911-10916.

Hill, N. I., Bailey, P. J., Griffiths, Y. M., \& Snowling, M. J. (1999). Frequency acuity and binaural masking release in dyslexic listeners. Journal of the Acoustical Society of America, 106(6), L53-L58.

Jeffries, S., \& Everatt, J. (2004). Working memory: Its role in dyslexia and other specific learning difficulties. Dyslexia, 10(3), $196-214$.

Margalit, M. (1997). Report of the committee for checking the abilities of children with learning disabilities. Ministry of Education.

Marshall, C. M., Snowling, M. J., \& Bailey, P. J. (2001). Rapid auditory processing and phonological ability in normal readers and readers with dyslexia. Journal of Speech, Language, and Hearing Research, 44(4), 925-940.

Meyler, A., \& Breznitz, Z. (2005). Visual, auditory and cross-modal processing of linguistic and nonlinguistic temporal patterns among adult dyslexic readers. Dyslexia, 11(2), 93-115.

Mody, M., Studdert-Kennedy, M., \& Brady, S. (1997). Speech perception deficits in poor readers: Auditory processing or phonological coding? Journal of Experimental Child Psychology, 64(2), 199-231.

Ram-Tsur, R., Faust, M., \& Zivotofsky, A. Z. (2006). Sequential processing deficits of reading disabled persons is independent of inter-stimulus interval. Vision Research, 46, 3949-3960.

Ram-Tsur, R., Faust, M., \& Zivotofsky, A. Z. (2008). Poor performance on serial visual tasks in persons with reading disabilities: Impaired working memory? Journal of Learning Disabilities, 41(5), 437-450.

Ramus, F., Rosen, S., Dakin, S. C., Day, B. L., Castellote, J. M., White, S., \& Frith, U. (2003). Theories of developmental dyslexia: Insights from a multiple case study of dyslexic adults. Brain, 126(Pt 4), 841-865.

Reed, M. A. (1989). Speech perception and the discrimination of brief auditory cues in reading disabled children. Journal of Experimental Child Psychology, 48(2), 270-292.

Reid, A. A., Szczerbinski, M., Iskierka-Kasperek, E., \& Hansen, P. (2007). Cognitive profiles of adult developmental dyslexics: Theoretical implications. Dyslexia, 13(1), 1-24.

Serniclaes, W., Sprenger-Charolles, L., Carre, R., \& Demonet, J. F. (2001). Perceptual discrimination of speech sounds in developmental dyslexia. Journal of Speech, Language, and Hearing Research, 44(2), 384-399.

Shankweiler, D., \& Crain, S. (1986). Language mechanisms and reading disorder: A modular approach. Cognition, 24(1-2), 139-168. 
Shatil, E. (1995a). One-minute test for pseudowords (Unpublished test, University of Haifa).

Shatil, E. (1995b). One-minute test for words (Unpublished test, University of Haifa).

Shaywitz, S. E. (1996). Dyslexia. Scientific American, 275, 98-104

Silani, G., Frith, U., Demonet, J. F., Fazio, F., Perani, D., Price, C., et al. (2005). Brain abnormalities underlying altered activation in dyslexia: A voxel based morphometry study. Brain, 128(Pt 10), 2453-2461.

Snowling, M., Bishop, D. V., \& Stothard, S. E. (2000). Is preschool language impairment a risk factor for dyslexia in adolescence? Journal of Child Psychology and Psychiatry, 41(5), 587-600.

Snowling, M. J. (2001). From language to reading and dyslexia. Dyslexia, 7(1), 37-46.

Tallal, P. (1980). Auditory temporal perception, phonics, and reading disabilities in children. Brain Lang, 9(2), 182-198.

Tallal, P., \& Piercy, M. (1973a). Defects of non-verbal auditory perception in children with developmental aphasia. Nature, 241(5390), 468-469.

Tallal, P., \& Piercy, M. (1973b). Developmental aphasia: Impaired rate of non-verbal processing as a function of sensory modality. Neuropsychologia, 11(4), 389-398.

Temple, E. (2002). Brain mechanisms in normal and dyslexic readers. Current Opinions in Neurobiology, 12(2), 178-183.

Van Ingelghem, M., van Wieringen, A., Wouters, J., Vandenbussche, E., Onghena, P., \& Ghesquiere, P. (2001). Psychophysical evidence for a general temporal processing deficit in children with dyslexia. NeuroReport, 12(16), 3603-3607.

Vasic, N., Lohr, C., Steinbrink, C., Martin, C., \& Wolf, R. C. (2008). Neural correlates of working memory performance in adolescents and young adults with dyslexia. Neuropsychologia, 46(2), 640-648.

Vellutino, F. R., Fletcher, J. M., Snowling, M. J., \& Scanlon, D. M. (2004). Specific reading disability (dyslexia): What have we learned in the past four decades? Journal of Child Psychology and Psychiatry, 45(1), 2-40.

Wechsler, D. (1997). Weschsler adult intelligence scale-III. San Antonio, T. X.: The Psychological Corporation.

Witton, C., Stein, J. F., Stoodley, C. J., Rosner, B. S., \& Talcott, J. B. (2002). Separate influences of acoustic AM and FM sensitivity on the phonological decoding skills of impaired and normal readers. Journal of Cognitive Neuroscience, 14(6), 866-874.

Wright, B. A., Bowen, R. W., \& Zecker, S. G. (2000). Nonlinguistic perceptual deficits associated with reading and language disorders. Current Opinions in Neurobiology, 10(4), 482-486. 